ИЗВЕСТИЯ АКАДЕМИИ НАУК ЭСТОНСКОИ ССР. ФИЗИКА МАТЕМАТИКА PROCEEDINGS OF THE ACADEMY OF SCIENCES OF THE ESTONIAN SSR. PHYSICS * MATHEMATICS

$1987,36,3$

\title{
ИЗМЕРЕНИЕ СПИНОВОЙ ПОЛЯРИЗАЦИИ И РЕЛАКСАЦИИ С ПОМОЩЬЮ ТЕХНИКИ ФАРАДЕЕВСКОГО ВРАЩЕНИЯ
}

\author{
(Представил Э. Липпмаа)
}

\begin{abstract}
Описан метод нерезонансного измерения электронной спиновой поляризации в прозрачных средах по вращению плоскости поляризации просвечивающего лазерного луча. Помехоустойчивая двухканальная оптическая система регистрации с амплитудно-цнфровым преобразованием фильтрованного фотоэлектрического сигнала и обработкой на мини-ЭВМ обеспечивает временное разрешение $10^{-5}$ c. Измерено изменение спиновой поляризаций парамагнитных $\mathrm{F}^{+}$-центров в аддитивно окрашенных кристаллах $\mathrm{CaO}$ при инверсии продольного магнитного поля со скоростью $d H_{z} / d t \approx 10^{6} \cdot \Gamma \mathrm{c} \cdot \mathrm{c}^{-1}$ после предварительного насыщения поляризации в поле $H_{2} \approx 25 \mathrm{\kappa}$ с при температуре $T \approx 0,5 \mathrm{~K}$.
\end{abstract}

\section{1. Введение}

Магнитооптические нерезонансные методы исследования спиновой динамики в твердом теле имеют определенные преимущества перед СВЧ-методами, когда измерения необходимо провести в непостоянном продольном магнитном поле (см., напр., $\left.\left[{ }^{1}\right]\right)$. Для измерения спин-решеточной релаксации используется как магнитный циркулярный дихроизм, так и фарадеевское вращение плоскости поляризации проходящего через объект света. Во втором случае возможна двухканальная регистрация без модуляции состояния поляризации измеряющего потока света, что выгодно для повышения временного разрешения $\left[{ }^{2}\right]$.

Ниже описана методика исследования спиновой поляризации парамагнитных центров окраски ионных кристаллов в сильно неравновесных условиях, но она может быть использована и при исследовании более широкого круга объектов, например, магнетизации и перемагнетизации прозрачных ферромагнитных сред [ $\left.{ }^{3}\right]$.

Во второй части нашей работы рассмотрена связь между углом фарадеевского вращения $\theta$ и степенью спиновой поляризации $P$ в основном состоянии центра окраски. Рассмотрено также влияние потери спиновой памяти на величину $P$ и вытекающие ограничения на интенсивность измеряющего света. В третьей части описана оптическая схема лазерного поляриметра и аппаратура регистрации временного хода $\theta$. Система управления экспериментом, регистрации и обработки данных базируется на мини-ЭВM «Aspect $3000 »$, входящей в состав спектрометра ионциклотронного резонанса CMC-47 (фирма «Bruker»). При этом используется стандартное программное обеспечение спектрометров ядерного магнитного резонанса. В четвертой части дана характеристика магнитной системы на базе сверхпроводящих соленоидов. Магниты позволяют создавать в области исследуемого объекта поляризующее магнитное поле порядка $H_{z}=30$ кГс, что при температуре $T \approx 0,5 \mathrm{~K}$ обеспе- 
чивает 99,9\% поляризацию электронных спинов (низкая температура получается откачкой паров над жидким изотопом $\left.{ }^{3} \mathrm{He}\right)$. Путем переключения тока одного соленоида в RC-цепь можно достичь скачкообразного изменения поля, в том числе прохождения через нуль со скоростью более $10^{6} \Gamma \mathrm{c} \cdot \mathrm{c}^{-1}$. В последней части работы в качестве иллюстрации приведены записи изменения угла вращения при инверсии магнитного поля, когда в качестве оптически активной среды использовался кристалл $\mathrm{CaO}$ с $\mathrm{F}^{+}$-центрами.

\section{2. Эффект Фарадея в парамагнитных центрах окраски}

Магнитной оптической активности кристаллов с парамагнитными центрами посвящены обзоры (см., напр., $[1,4,5])$; специфика центров окраски рассмотрена в $\left[{ }^{6}\right]$.

Угол вращения плоскости поляризации линейно-поляризованного света частоты $\omega=2 \pi / \lambda$ равен (в радианах)

$$
\theta(\omega)=\left[n_{r}(\omega)-n_{l}(\omega)\right] \omega d / 2 c,
$$

где $d-$ толщина объекта, $c-$ скорость света в вакууме. $\theta$ пропорционален разности показателей преломления для право- и левовращающихся компонентов циркулярной поляризации. В спектральной области, где находятся полосы поглощения парамагнитных центров, $\theta(\omega)$ имеет минимум, глубина которого пропорциональна разности энергий оптических переходов для правой и левой циркуляции, возникающей в продольном магнитном поле $H_{z}$. Точное количественное соотношение между $\theta(\omega)$ и $H=H_{z}$ зависит от природы (вырождения) энергетических уровней центра, величины взаимодействий (спин-орбитальной, электронноколебательной, зеемановской) и не будет здесь рассмотрено. Существенно, что суммарный эффект может быть составлен, во-первых, из температурно-независимой (диамагнитной) части $\theta_{D}(H)$, включающей фон от высокоэнергетических оптических переходов, а также результат зеемановского расщепления на энергию переходов между уровнями в центре, и, во-вторых, из температурно-зависимой (парамагнитной) части $\theta_{P}(H, T)$, обусловленной изменением заселенностей подуровней основного состояния центра

$$
\theta=\theta_{D}(H)+\theta_{P}(H, T) .
$$

Рассмотрим $S=1 / 2$ центры, где основное состояние представляет собой крамерсов дублет. Обозначим через $n^{-}$и $n^{+}$заселенности спиновых подуровней $|-1 / 2\rangle$ и $|+1 / 2\rangle$. Степень спиновой поляризации

$$
P=\frac{\left\langle S_{z}\right\rangle}{S}=\frac{n^{-}-n^{+}}{n^{-}+n^{+}} .
$$

Рассматриваемый в этой работе метод основан на пропорциональности между $\theta_{P}$ и $P$, которая сохраняется в изменяющемся во времени поле или при непостоянной температуре, когда $P$ отличается от своего равновесного значения

$$
P_{0}=\tanh \left(g \mu_{B} H / 2 k T\right) .
$$

Здесь $g \approx 2$ - спектроскопический фактор расщепления основного состояния, $\mu_{B} \equiv e h / 2 m_{\mathrm{e}}=9,274 \cdot 10^{-28}$ Дж.Гc $\mathrm{c}^{-1}$ - магнетон Бора, $k=$ $=1,381 \cdot 10^{-23}$ Дж. $\mathrm{K}^{-1}-$ постоянная Больцмана. Если $P$ выведено из равновесия (но не очень сильно), то $P$ (и $\theta_{P}$ ) начинают приближаться K равновесному значению по экспоненциальному закону

$$
P(t)=P_{0}+\left(P_{i}-P_{0}\right) \exp \left(-t / \tau_{1}\right),
$$


где $P_{i}$ - начальное значение $P, \tau_{1}-$ время спин-решеточной релаксации,

Экспериментальное различение $\theta_{P}$ и $\theta_{D}$ основано на том, что $\theta_{D}$ пропорционально полю, следует полю безынерционно и не зависит от температуры. Коэффициент пропорциональности между $\theta_{D}$ и $H$ может быть установлен из полевой зависимости $\theta(H)$ в условиях, когда $\theta_{P}$ постоянно: 1) при высоких температурах, когда $\mu_{B} H \ll k T, P_{0}=0$ и $\theta_{P}=0$, или 2) при низких температурах в сильном поле, когда $\mu_{B} H \gg$ $\gg k T, P_{0}= \pm 1$ и $\theta_{P}= \pm \theta_{P M}$ имеет максимальное абсолютное значение. Магнитооптическое вращение в окнах аппаратуры имеет диамагнитную природу и может быть включено в $\theta_{D}$. Далее в тексте под $\theta$ везде подразумевается $\theta_{P}$.

Известно, что интенсивное облучение центров окраски светом изменяет спиновую поляризацию: циркулярно-поляризованный свет может создавать поляризацию, а линейно-поляризованный уменьшать ее из-за потери спиновой памяти в возбужденном состоянии [ $\left.{ }^{7}\right]$. Типичное значенйе вероятности переворота спина в F-центре щелочно-галоидного кристалла в одном цикле накачки (возбуждение в результате поглощения кванта, колебательная релаксация, испускание кванта и релаксация в основном состоянии) составляет $\varepsilon \approx 0,1\left[{ }^{8}\right]$. Рассмотрим ограничение на интенсивность измеряющего света, вытекающее из явления потери спиновой памяти.

Пусть мы имеем кристалл толщиной $d$ (см), содержащий $N$ парамагнитных центров $\left(\mathrm{cm}^{-3}\right)$, с сечением захвата центра на длине волны измерения $\sigma\left(\mathrm{cm}^{2}\right)$. Если на переднюю плоскость кристалла падает $n_{0}$ фотонов $\left(\mathrm{cm}^{-2} \cdot \mathrm{c}^{-1}\right)$, то без учета отражения мы имеем на глуб́ин $z$ $n_{0} \exp (-\sigma N z)$ фотонов и $\varepsilon n_{0} N \exp (-\sigma N z)$ актов переворота спина, каждый из которых изменяет на 2 величину разности $n=n^{-}-n^{+}=N P$. Следовательно, если мы имеем в начале облучения равновесную спиновую поляризацию $P_{0}$, то она начинает в ходе облучения уменьшаться со скоростью $\tau_{1}^{-1}+\tau_{p}^{-1}$, где

$$
\tau_{p}^{-1}(z)=2 \varepsilon n_{0} \sigma \exp (-\sigma N z) .
$$

Установившееся (зависящее от глубины z) значение спиновой поляризации при $t \rightarrow \infty$

$$
P_{\infty}(z)=\frac{\tau_{1}^{-1}}{\tau_{1}^{-1}+\tau_{p}^{-1}(z)} P_{0}
$$

Вместо угла вращения

$$
\theta_{0}=\beta \sigma N d \cdot P_{0}
$$

которую мы имели бы в отсутствие деполяризации, имеем

$$
\theta_{\infty}=\beta N \sigma \int_{0}^{d} P_{\infty}(z) d z
$$

Требуем, чтобы деполяризация была ограничена, так что относительное уменьшение угла поворота (или поляризации) была меньше заданного малого числа $\eta \ll 1$ :

$$
\left(\theta_{0}-\theta_{\infty}\right) / \theta_{0}<\eta .
$$

Интегрированием (9), с учетом (6) и (7), получим

$$
n_{0}<\frac{\exp (\sigma N d \cdot \eta)-1}{1-\exp (-\sigma N d(1-\eta))} \cdot \frac{\tau_{1}^{-1}}{2 \varepsilon \sigma}
$$


Если кристалл оптически толстый, а условие на $\eta$ сильное, так что

$$
\sigma N d \gg 1 \gg \eta \sigma N d \text {, }
$$

TO

$$
n_{0}<\frac{N d \tau_{1}^{-1}}{2 \varepsilon} \cdot \eta
$$

Если кристалл оптически тонкий, то

$$
n_{0}<\frac{\tau_{1}^{-1} \cdot \eta}{2 \varepsilon \sigma}
$$

Если $\tau^{p-1} \gg \tau_{1}^{-1}$, то ограничено общее число фотонов, падающих на кристалл во время измерения $\Delta t$

$$
n_{0} \Delta t<\frac{N d \cdot \eta}{2 \varepsilon[1-\exp (-\sigma N d)]} \approx \frac{N d}{2 \varepsilon} \cdot \eta .
$$

Пусть мы имеем кристалл, содержащий $N=10^{17}$ центров в $1 \mathrm{~cm}^{3}$, $\sigma=3 \cdot 10^{-16} \mathrm{~cm}^{2}$ (типично для центров окраски), $d=0,1 \mathrm{cм}$, так что в кристалле поглощается $95 \%$ из числа $n_{0} \cdot \Delta t$. Потребуем, чтобы $\eta=10^{-2}$. Предположив $\varepsilon=0,05$, получим $n_{0} \Delta t<10^{15}$ фотонов на $1 \mathrm{~cm}^{2}$.

\section{3. Регистрация временного хода фарадеевского вращения}

В литературе описаны установки определения $\theta$, где в зависимости от цели упор делается либо на точном измерении угла $\left(10^{-6}-10^{-7}\right.$ град; cм. $\left.\left[{ }^{9-11}\right]\right)$, как, например, при наблюдении несохранения четности в атомной спектроскопии $\left[{ }^{11}\right]$, либо на быстрой регистрации $\left(10^{-6}\right.$ $10^{-7}$ c) скачков угла между двумя значениями, различающимися между собой на несколько градусов, например, при оптическом считывании информации $\left[{ }^{3}\right]$. При регистрации спиновой динамики необходим компромисс между временным и угловым разрешениями.

Наша установка представляет собой лазерный поляриметр с двухканальной одновременной регистрацией интенсивности двух проекций проходящего пучка на взаимно перпендикулярные направления поляризации, с последующим аналого-цифровым преобразованием фильтрованного фотоэлектрического сигнала и машинной обработкой информации (рис. 1). С помощью трех зеркал $3,5,6$ свет лазера 1 направляется через диспергирующую призму 2 и ослабляющие светофильтры 7 на кварцевый клин 8 , в котором происходит разветвление пучка на два линейно-поляризованных в перпендикулярных плоскостях. Отобранный пучок проходит ряд окон из плавленого кварца 9,11 , $13-16$, часть из которых находится внутри сверхпроводящего магнита 10 , а также охлаждаемый

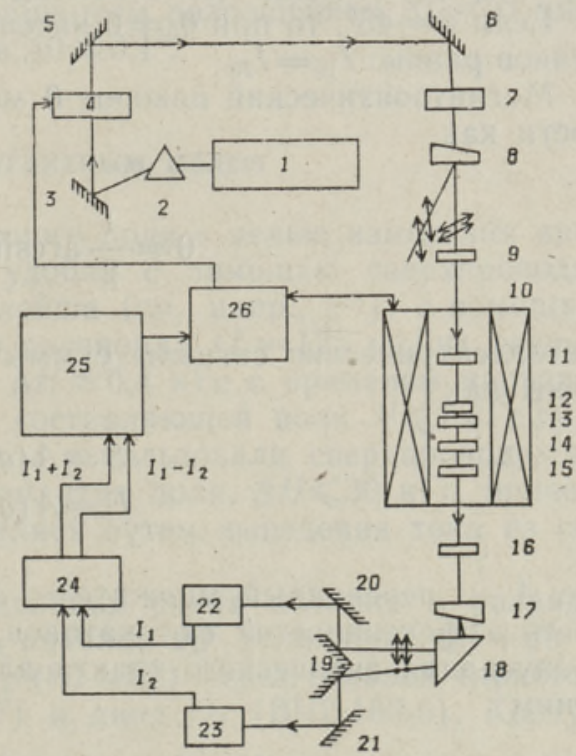

Рис. 1. Схема экспериментальной установки. 
объект 12, и снова разветвляется на два пучка во втором кварцевом клине 17 , имеющим ось под углом $\varphi \approx 45^{\circ}$ к оси клина 8 . Два пучка перпендикулярной поляризации, расходящиеся под углом $\approx 2^{\circ}$, направляются с помощью системы из призмы полного отражения 18 и зеркал $19-21$ на катоды двух фотоумножителей ФЭУ-79 22, 23, находящихся внутри магнитного экрана из мягкого железа. Фотоэлектрические сигналы тока $I_{1}$ и $I_{2}$ поступают в преобразователь тока в напряжении 24 , из которого сигналы, пропорциональные сумме и разности токов, поступают в мини-ЭВM «Aspect $3000 » 25$, осуществляющий накопление и обработку данных, а также управление электромеханическим прерывателем света 4 и блоком питания магнита 26. Оптическая система предусмотрена для работы в диапазоне длин волн от ближнего ультрафиолета до ближнего инфракрасного, поэтому преломляющие элементы изготовлены из кварца.

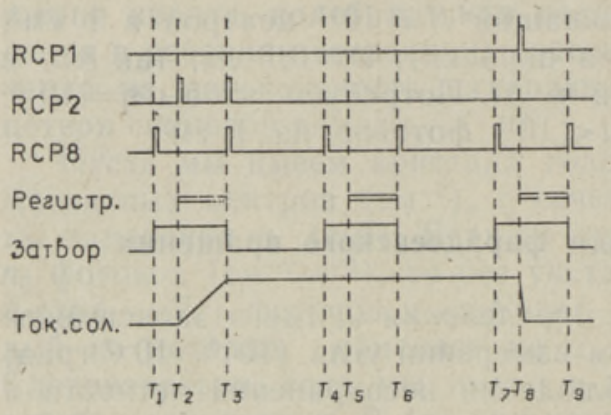

Рис. 2. Временная диаграмма управляю- щих сигналов.

мого переключением тока соленоида.

Если $\varphi=45^{\circ}$, то при $\theta_{0}=0$ интенсивности выходящих из анализатора пучков равны: $J_{10}=J_{20}$.

Магнитооптический поворот $\theta$ может быть выражен через интенсивности как

$$
\theta=\frac{1}{2} \arcsin \frac{J_{1}-J_{2}}{J_{1}+J_{2}} .
$$

Фотоэлектрические сигналы суммы $S$ и разности $D$ могут быть выражены как

$$
\begin{aligned}
& S=A\left(q J_{1}+J_{2}\right), \\
& D=A\left(q J_{1}-J_{2}\right),
\end{aligned}
$$

где $A$ - переводный множитель, $q$ - множитель, учитывающий разность эффективностей фотокатодов, коэффициентов усиления, а также пропускания оптического тракта для двух каналов. Обращая (17), получим

$$
\frac{J_{1}-J_{2}}{J_{1}+J_{2}}=\frac{(q-1) S+(q+1) D}{(q+1) S+(q-1) D},
$$


где

$$
q=\frac{S_{0}+D_{0}}{S_{0}-D_{0}}
$$

находится из калибровочного измерения при $\theta_{0}=0$.

Для быстрого вычисления $\theta$ из измеренных значений $D$ и $S$ налажена программа на языке Паскаль с организацией виртуальной памяти для преобразования больших массивов данных в сравнительно малом объеме памяти. Для быстрых расчетов с плавающей запятой создана подпрограмма на языке Ассемблер. Функция arcsin табулирована. Программа на языке Паскаль может работать подпрограммой системной ЯMP программы DISNMR в режиме деления временчи в ходе накопления данных.

Временное разрешение установки ограничено быстродействием 12-разрядного двухканального аналого-цифрового преобразователя (5 мкс на точку) и предусилителя (3 мкс).

При рассмотрении углового разрешения необходимо отметить, что в случае одновременной двухканальной регистрации погрешности, связанные с нестабильностью источника, а также с вибрацией элементов оптического тракта, в значительной мере компенсируются. Когда выполнено условие, что флуктуации фотоэлектрического сигнала больше разрешающей способности аналого-цифрового преобразователя (этого можно достичь, увеличивая напряжение на ФЭУ), то основным источником погрешности будут статистические флуктуации числа фотоэлектронов. Элементарное вычисление дает

$$
\Delta \theta=\frac{180^{\circ}}{\pi \sqrt{2 n^{\prime} L}},
$$

где $L-$ квантовый выход фотокатода, $n^{\prime}=n_{0} \exp (-\sigma N d) \cdot s-$ суммарное количество фотонов в двух каналах анализатора, $s$ - поперечное сечение пучка в объекте. Истинная погрешность несколько больше.

Примем ограничения на световой поток, как в примере в конце раздела $2\left(n_{0}<10^{15} \mathrm{~cm}^{-2}\right)$ и $s=0,1 \mathrm{~cm}^{2}, L=0,1$. Тогда при регистрации в течение 1 с некоторого процесса с временным разрешением $\Delta t=10$ мкс, получим, что в каждом из $10^{5}$ каналов $\Delta \theta \approx 0,1^{\circ}$.

\section{4. Управление магнитным полем}

Скачкообразное изменение магнитного поля с целью измерения времени спин-решеточной релаксации удобно с помощью сверхпроводящего магнита. В работах Х. Кальбфлейша (см., напр., $\left[{ }^{12}\right]$ ) с помощью малоиндуктивного сверхпроводящего соленоида ( $L=125$ мкГн) осуществлялись скачки продольного поля $\Delta H \approx 0,4$ кГс с временем нарастания $\Delta t \approx 1$ мкс на фоне постоянной составляющей поля $H \leqslant 7$ кГс другого источника. В отличие от $\left.{ }^{12}\right]$ мы использовали сверхпроводящий соленоид для получения больших амплитуд поля, $\Delta H \leqslant 30$ кГс, причем быстрые изменения поля осуществлялись путем выведения тока из соленоида.

На рис. 3 изображена принципиальная схема питания и разряда соленоида. Ток основного источника питания $I_{1}{ }^{*}$ величиной до $+90 \mathrm{~A}$ питает сверхпроводящий соленоид $L_{1}(80 \mathrm{~m} \Gamma$ н) через силовой тиристор высокого напряжения $D_{1}$ (ТБ3-200-7) и диод $D_{4}$ (ВЧ2-160-6). Контур

* Использовался блок питания СПС-150 Специального конструкторского бюро АН ЭССР с некоторыми модификациями. 
из $C_{2}\left(400\right.$ мкФ), $L_{2}\left(0,5\right.$ мкГ), $R_{2}(22$ кОм $), D_{2}$ (ТЧ63-7) и $D_{3}$ (ВЛ10-8) служит для выключения тиристора $D_{1}$. При подаче на вход $A$ управ ляющего сигнала (RCP1) ток соленоида переключается на контур гашения тока из $C_{1}\left(0 \ldots 700\right.$ мкФ), $R_{1}\left(0,242 \ldots 8\right.$ Ом) и $D_{6}$ (ВЧ2-160-6). Дополнительный источник тока $I_{2}$ (блок Б5-43, ток до -2 А) был подключен через диод $D_{5}$ (ВЛ10-8) в тех случаях, когда реверсия магнитного поля осуществлялась методом обращения тока соленоида.

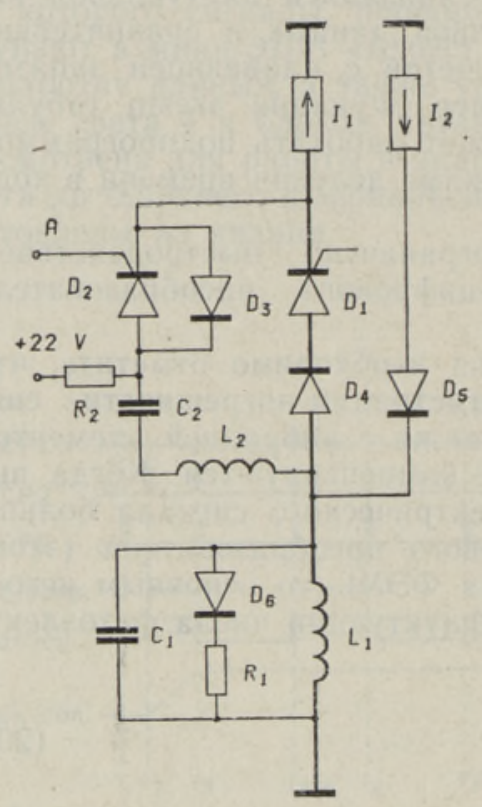

Рис. 3. Схема питания соленоида.

Альтернативный метод реверсии магнитного поля заключается в использовании двух магнитов. В качестве источника конечного поля использовался сверхпроводящий магнит ЯМР-спектрометра СXР-200, работающий в короткозамкнутом режиме с пониженным полем (несколько кГс вместо номинального значения $47 \mathrm{\kappa} Г \mathrm{c}$ ). Внутри теплого отверстия Брукеровского магнита находится магнитооптический криостат с управляемым соленоидом $L_{1}$, расположенным соосно и концентрически по отношению к первому магниту. Благодаря значительному различию размеров соленоидов большинство силовых линий внутреннего соленоида замкнуты внутри внешнего соленоида и индуцированные во внешнем соленоиде токи относительно мало сказываются на поле в центре. Преимуществом такой схемы перед схемой с обращением тока в одном соленоиде является то, что переход соленоида в нормальное состояние в момент переключения тока не препятствует проведению измерений.

Параметры контура гашения тока выбираются близкими к критическим $\left(R_{1} \approx\left(L_{1} / 4 C_{1}\right)^{1 / 2}\right)$. Максимальная скорость изменения поля $d H / d t \approx 10^{3} \mathrm{\kappa} \Gamma \mathrm{c} \cdot \mathrm{c}^{-1}$ в основном ограничена индуктивным напряжением $U=L \cdot d I / d t \leqslant 700 \mathrm{~B}$.

\section{5. Пример: $\mathrm{F}^{+}$-центры в $\mathrm{CaO}$}

$\mathrm{B}$ качестве примера рассмотрим поведение $\mathrm{F}^{+}$-центров в монокристалле $\mathrm{CaO}$ при инверсии магнитного поля. $\mathrm{F}+$-центр в окислах типа $\mathrm{CaO}$ представляет собой анионную вакансию, в которой локализован один электрон (см. $\left.\left[{ }^{13}\right]\right)$. Основное состояние этого центра - крамерсов дублет. Фарадеевское вращение в электронно-колебательной $\mathrm{F}^{+}$-полосе поглощения, имеющей максимум при 3,65 эВ, изучено В $\left[{ }^{14,15}\right]$.

В наших опытах использовался монокристалл $\mathrm{CaO}$ фирмы «W\&C Spicer Ltd» чистоты 3N5, имеющий желтую окраску. Содержание F-центров (2 электрона в анионной вакансии) по поглощению не менее $10^{18} \mathrm{~cm}^{-3}$, содержание $\mathrm{F}^{+}$-центров, оцененное по парамагнитному вращению, около $10^{17} \mathrm{~cm}^{-3}$. Для измерений из кристалла была выколота плоскопараллельная пластинка с $d=0,047$ см, которая в атмосфере сухого гелия была герметически закрыта в кварцевую ампулу со склеянными окнами. Ампула с кристалџом охлаждалась в магнито-оптическом криостате с помощью жидкого изотопа ${ }^{3} \mathrm{He}$ до температуры $T=0,5 \mathrm{~K}$. Измерения проводились линией аргонного ионного лазера COHERENT CR 12 с $\lambda=335,8$ нм, которая попадает в экстремум спектральной зависимостй 


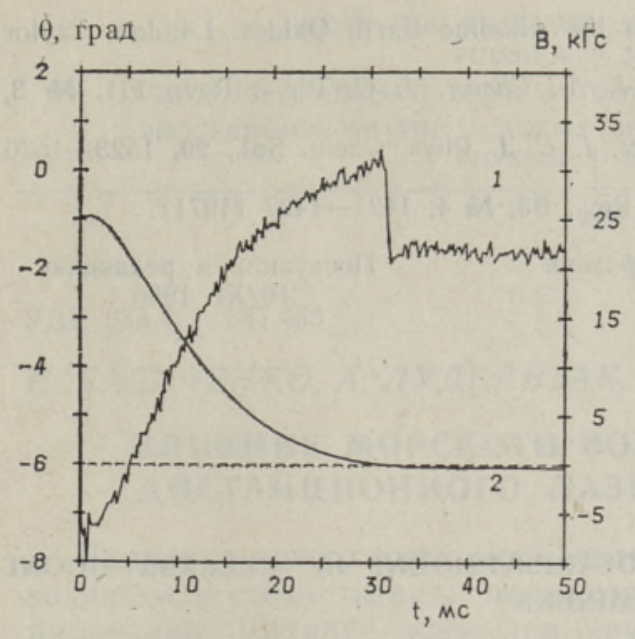

Рис. 4. Поворот Фарадея в СаO при $\lambda=335,8$ нм и $T<0,65 \mathrm{~K}(1)$ во время инверсии магнитного поля (2).

Рис. 5. Участок кривой $I$ рис. 4 с временным разрешением 12 мкс.

$\theta$, град

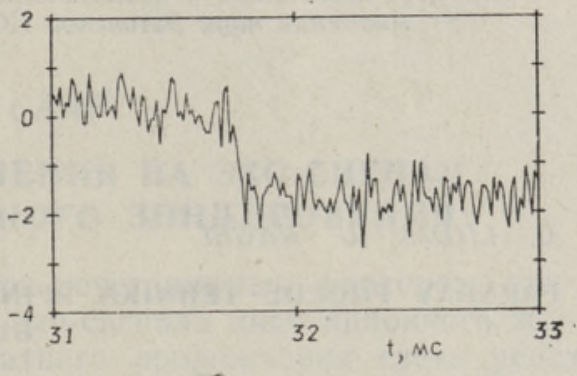

парамагнитного вращения $\left[{ }^{14,15}\right]$. Падающий на кристалл поток был $n \approx 10^{13}$ фотонов $\cdot \mathrm{c}^{-1}$.

На рис. 4 изображена временная зависимость угла $\theta$, измеряемая во время реверсии магнитного поля с начального значения $+25,4$ кГс по конечное - 0,69 кГс. Поле измерено с помощью полупроводникового датчика Холла в аналогичном опыте. Перед разрядом $P_{0} \geqslant 0,99$. Основное изменение $\theta$ во время изменения поля обусловлено диамагнитным эффектом. Скачок при пересечении нулевого поля есть парамагнитный эффект, его величина $\Delta \theta=2^{\circ}$ численно равна максимальному парамагнитному вращению $\theta_{P M}$ в этом кристалле.

Регистрация сигнала производилась с временным разрешением $\Delta t=12$ мкс, однако с целью сглаживания кривой перед построением графика на рис. 4 осуществлялось суммирование по 16 точкам. На рис. 5 изображен участок из оригинальной записи, содержащей скачок при отметке $t=31,77$ мс. Учитывая, что в этот момент скорость изменения поля $d H_{z} / d t=109 \mathrm{k} \Gamma \mathrm{c} \cdot \mathrm{c}^{-1}$, получим, что скачок $\theta$ имеет место в интервале $\Delta H_{z}<2$ Гс. Эта величина представляет собой разброс поперечных полей в данном кристалле СаО. Отметим, что эта величина на порядок меньше ширины линии оптически детектируемого с помощью Фарадеевского вращения магнитного резонанса $\mathrm{F}^{+}$-центров на частоте 35 ГГц $\left[{ }^{14}\right]$, и соответствует ширине линии ЭПР, детектируемой при 9 ГГц с помощью магнитного циркулярного дихроизма $\left[{ }^{16}\right]$, но на 2 порядка больше ширины линий ӘПР на радиочастоте [14].

\section{ЛИТЕРАТУ РА}

1. Запасский В. С., феофилов П. П. УФН, 116, № 1, 41-78 (1975).

2. Rabson, T. A. Appl. Opt., 5, № 8, 1293-1296 (1966).

3. Chen, D., Otto, G. N., Schmit, F. M. IEEE Trans. Magn. MAG-9, № 2, 66-83 (1973).

4. Старостин Н. В., Феофилов П. П. УФН, 97, № 4, 621-655 (1969).

5. Buckingham, A. D., Stephens, P. J. Ann. Rev. Phys. Chem., 17, 399 (1966).

6. Mort, J., Lüty, F., Brown, F. C. Phys. Rev., 137, № 2A, 566-573 (1965).

7. Mollenauer, L. F., Pan, S. Phys. Rev., B6, № 3, 772-787 (1972).

8. Лийдья Г., Соовик T. ФТТ, 21, № 8, 2377-2383 (1979).

9. Александров Е. Б., Запасский В. С. Опт. и спектр., 41, № 5, 855-858 (1976).

10. Запасский В. С. Опт, и спектр., 52, № 6, 1105-1108'(1982).

11. Хриплович И. Б. Несохранение четности в атомных явлениях. М., «Наука», 1981.

12. Becher, W., Kalbfleisch, H. Phys. Status Solidi (a), 2, № 1, 109-113 (1970). 
13. Henderson, B., Wertz, J. E. Defects in the Alkaline Earth Oxides. London, Taylor \& Francis Ltd., 1977.

14. Kemp, J. C., Ziniker, W. M., Glaze, J. A., Cheng, J. C. Phys. Rev., 171, № 3, $1024-1036$ (1968).

15. Bessent, R. G., Cavenett, B. C., Hunter, I. C. J. Phys. Chem. Sol., 29, 1523-1530 (1968).

16. Merle d'Aubigné, Y., Roussel, A. Phys. Rev., B3, № 4, 1421-1427 (1971).

Ннститут химической и биологической физики Академии наук Эстонской ССР

Поступила в редакцию $10 / \mathrm{XI} 1986$

G. LIIDJA, U. NAGEL

\section{FARADAY POORDE TEHNIKA SPINNPOLARISATSIOONI JA -RELAKSATSIOONI UURIMISEKS}

Metoodikat elektronide spinnpolarisatsiooni mõõtmiseks läbipaistvates keskkondades laserikiire polarisatsioonitasandi pöörde kaudu on arendatud värvitsentrite uurimiseks tugevasti mittetasakaalulistes olukordades hea aeglahutusega. Süsteemi iseärasused on ülikõrgsagedusvälja puudumine, fotoelektrilise signaali üheaegne mõõtmine kahes kanalis (see välistab vajaduse laserikiire polarisatsiooni moduleerimiseks), magnetvälja kiired ( $10^{5}$ gaussi sekundis) ja suure amplituudiga muutumised ning madal temperatuur $(\approx 0,5 \mathrm{~K})$ täieliku spinnpolarisatsiooni saavutamiseks. Andmetöötluseks ja eksperimendi juhtimiseks kasutatakse miniarvutit «Aspect 3000».

G. LIIDJA, U. NAGEL

\section{SPIN POLARIZATION AND RELAXATION MEASUREMENTS BY FARADAY ROTATION TECHNIQUE}

Rotation of the plane of polarization of light by paramagnetic defects in transparent solids is useut to measure the electron spin polarization of defects (color centers) under strongly nonequilibrium conditions. Starting with nearly 100 per cent spin polarization in a magnetic field of about 30 kiloGauss at subKelvin temperatures, the field is altered with the rate up to $10^{6}$ gauss per second, by discharging the superconducting solenoid into a RC circuit, or even reversed. To achieve good time resolution, a two channel compensation technique is applied in photoelectric measurements instead of beam modulation. The computer «Aspect 3000» and standard software of «Bruker» NMR spectrometers are used together with some special programs to compute the angle of rotation and to display its time evolution. The time resolution of the method is mainly limited by the analog/digital converter, the angle resolution by photon statistics and the incident beam intensity by light induced spin depolarization (spin memory loss) in color centers. A rapid change of the paramagnetic rotation angle is detected with $\mathrm{F}^{+}$centers in the $\mathrm{CaO}$ crystal when crossing the zero field. 